\title{
Sojaextrakt - eine Option in der Dermokosmetik
}

\section{Zusammenfassung}

Aufgrund hormoneller Veränderungen in der Postmenopause und zusätzlicher extrinsischer Faktoren wie UV-Strahlung und oxidativem Stress werden in der alternden Haut eine zunehmende Atrophie der Epidermis und eine dermale Atonie beobachtet. Maßnahmen zur Verzögerung der Hautalterung haben bei Frauen in reifem Lebensalter daher einen hohen Stellenwert. Zwei kontrollierte Studien belegen die positiven Effekte sojaextrakthaltiger Pflegepräparate auf die Haut: Die Dichte der reifen Haut wird verbessert, die Faltenbildung an Körper- und Gesichtshaut signifikant reduziert.

\section{Abstract}

Hormonal changes in postmenopause and additional extrinsic factors cause an increasing atrophy of the epidermis and dermal atonie in aging skin. Therefore measures to retard skin aging are of outmost importance for women. Two double-blind studies show the positive effects of cremes containing soyextract on skin: densitiy of mature skin will be improved, wrinkling of body and face significantly reduced.

\section{Einleitung}

Die alternde Haut ist durch zunehmende Schlaffheit, Xerose und Atrophie charakterisiert. Bereits kurz nach der Menopause ist bei Frauen eine Abnahme der Epidermisdicke um bis zu 50\% und der Dermisdicke um bis zu 30\% festzustellen, da der stimulierende Effekt von Östrogenen auf die Zellteilung entfällt. Typisch für die Altershaut ist darüber hinaus eine durch den Untergang von Melanozyten bedingte Blässe.

Eine der wichtigsten Säulen bei der Prävention der Hautalterung ist der effektive Schutz vor UV-Strahlung, die als Hauptfaktor für ein vorzeitiges Photo-Aging verantwortlich ist. Darüber hinaus existiert jedoch mittlerweile eine Vielzahl topischer Substanzen zur Verlangsamung des Alterungsprozesses in der Haut und zur Reparatur altersbedingter Hautschäden. Hierzu gehören Vitamin-A-Präparate wie Retinol und Retinaldehyd, die bei ausreichend langer Anwendung über eine Zunahme des Kollagens im
Unterhautgewebe zu einer strafferen und glatteren Haut führen $[1,2]$. Auch Vitamin C und E sind heute als klassische Antioxidanzien zum Schutz vor freien Sauerstoffradikalen in vielen kosmetischen Produkten enthalten $[3,4]$. Als optimal hat sich die Kombination beider Vitamine herausgestellt, da durch Kooperation zwischen beiden Redoxsystemen eine Wirksteigerung erreicht wird. Ebenfalls bekannt und mittlerweile gut untersucht ist die antioxidative Wirkung von Sojaextrakten [5]. Die experimentellen und klinisch-kosmetologischen Erfahrungen mit sojaextrakthaltigen Externa sollen im Folgenden dargelegt werden.

\section{Wirkansatz von Sojaextrakten}

Zu den aus der Sojabohne extrahierten Wirksubstanzen gehören die Isoflavone. Es handelt sich um nicht-steroidale Substanzen, deren Anti-Aging-Effekte auf mehrere Wirkmechanismen zurückgeführt werden können. Sojaextrakte, die in diese Gruppe 
gehören, besitzen zum einen eine antioxidative Wirkung und schützen Zellen dadurch vor einer Lipidperoxidation. Sie hemmen außerdem Tyrosinkinasen, die als Bestandteil intrazellulärer Signalkaskaden eine wichtige Rolle bei Tumorzellproliferation und Metastasierung spielen [6]. Zudem üben Sojaextrakte auch einen stimulierenden Effekt auf die Hyaluronsäuresynthese aus und wirken so der im Alter zunehmenden Hauttrockenheit entgegen. Schließlich schützen diese Substanzen Kollagen vor der enzymatischen Degradation [7].

\section{Experimentelle Untersuchungen mit sojaextrakthaltigem Externum}

Erste Hinweise für Anti-Aging-Effekte von Sojaextrakten stammen aus experimentellen Untersuchungen an Hautmodellen. Danach führt die Behandlung kultivierter gealterter Haut mit einem sojaextrakthaltigen Externum zu einer ausgeprägten Stimulation der Kollagenneosynthese, die anhand des Einbaus von 14C-markiertem Prolin erfasst wurde [8]. Gleichzeitig wird ein Schutz von Matrixproteinen vor einer enzymatischen Degradierung erreicht. Aufgrund dieser Effekte ist im Modellsystem eine deutliche Zunahme an Kollagenfasern in der behandelten Haut zu erkennen, die in der unbehandelten Kontrolle ausbleibt. Strukturell zeigt die behandelte Haut damit wieder eine größere Ähnlichkeit zur nicht gealterten Normalhaut mit ihrem hohen Anteil an Kollagenfasern.

Ebenfalls positive Effekte werden aus einem weiteren Hautmodell nach Applikation eines sojaextrakthaltigen Externums, das zusätzlich Phloroglucin und Zimtsäure enthält (Novadiol ${ }^{\circledR}, \mathrm{Vi}$ chy-Cosmetique Active Deutschland), berichtet [9]. Der histologischen Auswertung zufolge führt die regelmäßige Applikation des Präparates zu einer Zunahme der zellulären Aktivität in der Epidermis und zu einer verbesserten Verzahnung von Ober- und Unterhautgewebe, sodass die epidermale Atrophie im Vergleich zur unbehandelten Haut erheblich reduziert werden kann.

\section{Ergebnisse kontrollierter Studien mit sojaextrakthaltigem Externum}

Bestätigt werden diese experimentellen Untersuchungen durch eine offene multizentrische Studie mit einem sojaextrakthaltigen Externum (plus Phloroglucin und Zimtsäure), die an acht europäischen Kliniken in Deutschland, Frankreich, Italien, Spanien und der Schweiz erstellt wurde [10]. An der Studie beteiligten sich 234 postmenopausale Frauen (Phototyp der Lichtempfindlichkeit II - III) im durchschnittlichen Alter von 56 Jahren. Keine der Probandinnen erhielt eine Hormonersatztherapie, sodass die Effekte des Prüfpräparates nicht durch Auswirkungen einer Östrogensubstitution auf die Haut beeinträchtigt wurden. Über 12 Wochen wurde zweimal täglich eine sojaextrakthaltige Creme (Novadiol ${ }^{\circledR}$, Vichy Cosmétique Active Deutschland) auf Gesicht, Nacken und einen Arm aufgetragen. Der andere Arm blieb unbehandelt und diente als Kontrolle.

Die Hautbeschaffenheit wurde zu Studienbeginn, nach vier- und nach zwölfwöchiger topischer Applikation der Creme bewertet. Für die Auswertung wurde einmal die klinische Untersuchung durch den Prüfarzt herangezogen, der Trockenheit, Rauigkeit, Falten und Atonie der Haut mittels eines Scoresystems (Skala von 0-9) bewertete. Zum anderen beurteilten auch die Probandinnen selbst den Behandlungserfolg.

Neben dieser subjektiven Bewertung erfolgte außerdem eine objektivierbare Beurteilung der Haut mit dem DensiScore ${ }^{\circledR}$, einem semiquantitiven Messgerät. Mit diesem Instrument wird ein definiertes Hautareal mit einer Standardkompression von $42 \%$ zusammengedrückt, sodass die erfasste Haut von ursprünglich $24 \mathrm{~mm}$ Breite auf $14 \mathrm{~mm}$ reduziert wird [11]. Alle Messungen werden an der Armaußenseite durchgeführt. Dabei sitzt die Probandin mit aufgestützten Unterarmen, damit der Trizeps relaxiert. Das im Messfeld des DensiScore ${ }^{\circledR}$ entstehende Faltenprofil wird dann mit einer sechsstufigen Skala von Referenzfotografien verglichen, bei der Grad 1 junger Haut und Grad 6 gealterter Haut entspricht (Abb.1).

In der Beurteilung durch den Arzt zeigte sich im Studienverlauf eine kontinuierliche Besserung von Rauigkeit, Trockenheit und Falten der behandelten Haut (Abb. 2). Am stärksten ausgeprägt war die Abnahme der Trockenheit in der Gesichtshaut mit 33\% bis Woche 12. Die Hautrauigkeit verringerte sich signifikant um bis zu $22 \%(\mathrm{p}<0,05)$; die Gesichtsfalten bildeten sich um $20 \% \mathrm{zu}-$ rück ( $p<0,05)$. Auch die für die postmenopausale Haut charakteristische Atonie nahm ab, und zwar ebenfalls signifikant um $24 \%$ $(p<0,05)$. Übereinstimmend mit den Arztbefunden beurteilten auch die Probandinnen den Effekt der sojaextrakthaltigen Creme auf die Haut eindeutig positiv. Bereits nach einmonatiger Applikation attestierten die Prüfpersonen eine günstige Beeinflussung des Hautbildes mit verbesserter Hydratation, Hautfestigkeit und Ausstrahlung.

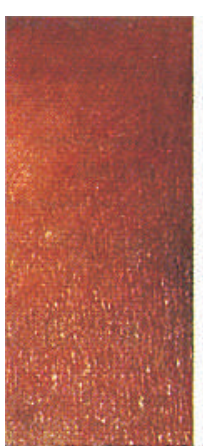

1

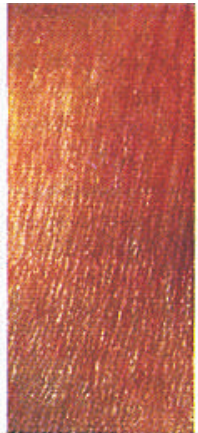

2

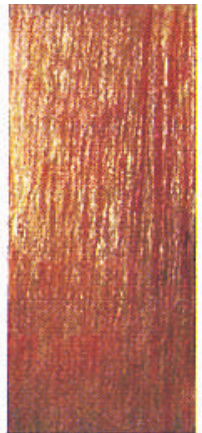

3

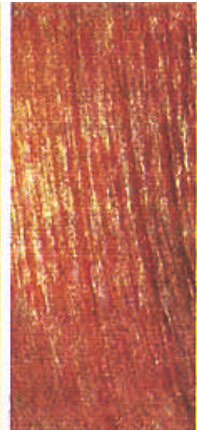

4

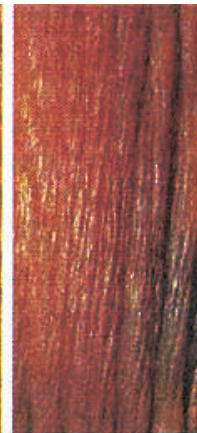

5

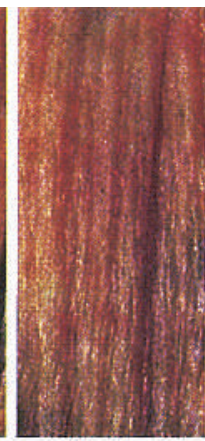

6
Abb. 1 Referenzfotografien zur Beurteilung des Hautfaltenprofils mit dem DensiScore ${ }^{\circledR}$ [11]. 

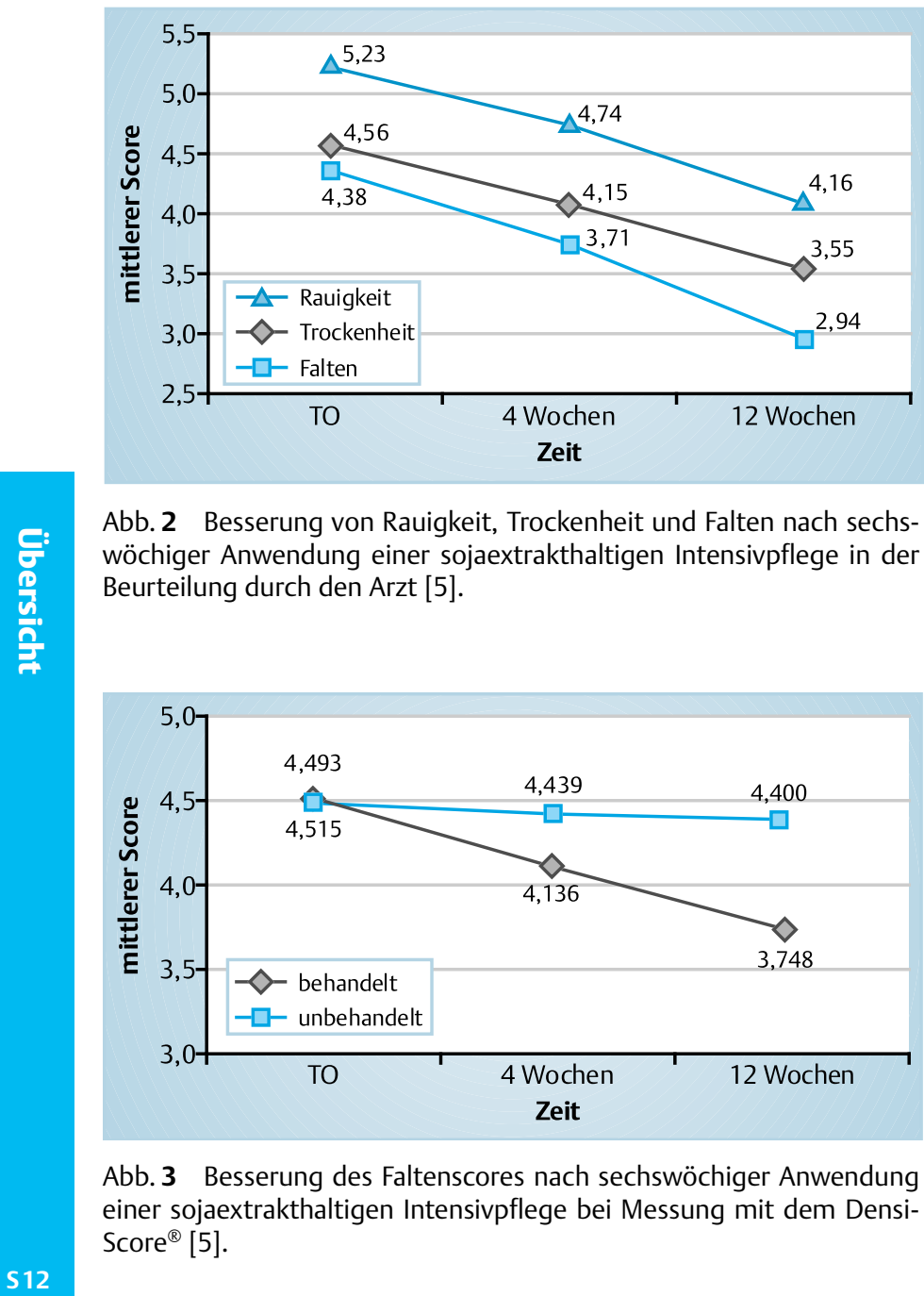

Abb. 2 Besserung von Rauigkeit, Trockenheit und Falten nach sechswöchiger Anwendung einer sojaextrakthaltigen Intensivpflege in der Beurteilung durch den Arzt [5].

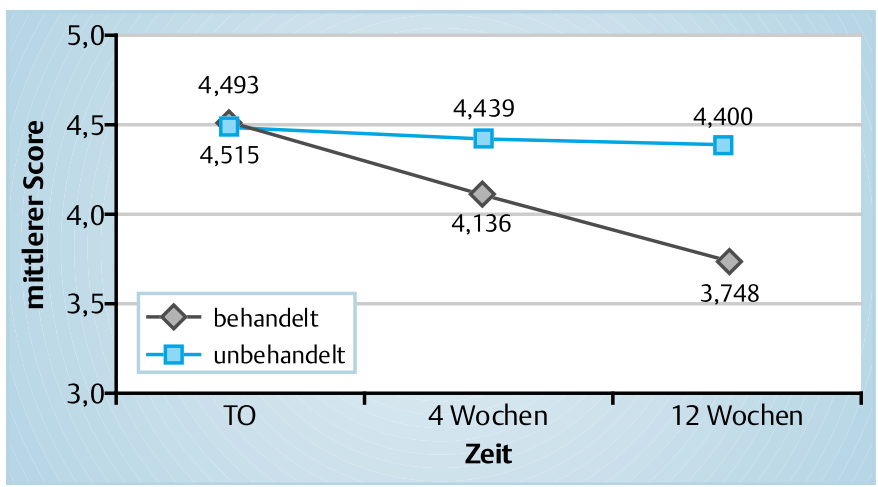

Abb. 3 Besserung des Faltenscores nach sechswöchiger Anwendung einer sojaextrakthaltigen Intensivpflege bei Messung mit dem DensiScore ${ }^{\circledR}[5]$.

Bei der Messung mit dem DensiScore ${ }^{\circledR}$ zeigte sich am unbehandelten Arm vom Studienstart bis zur zwölften Studienwoche praktisch keine Veränderung. Dagegen sank der Faltenscore auf dem behandelten Arm signifikant um durchschnittlich $17 \%$ im Vergleich zur Kontrolle $(\mathrm{p}<0,05)$. Die Besserung des Hautzustandes war bereits nach der vierten Behandlungswoche sichtbar und setzte sich bis Studienende fort (Abb. 3).

Vergleichbar positive Daten stammen auch aus einer kleineren offenen Studie an 45 postmenopausalen Frauen (Typ der Lichtempfindlichkeit II-III) im mittleren Alter von 57 Jahren, in der eine sojaextrakthaltige Körperlotion (Novadiol Körperlotion, Vichy Cosmétique Active Deutschland) evaluiert wurde [12]. Alle Frauen besaßen eine empfindliche Haut mit einem Hautfaltenscore von mindesten 3 im DensiScore ${ }^{\circledR}$ bei Studienbeginn. Die Hauttrockenheit am Bein wurde anhand der Referenzskala mit mindestens 2 bewertet. Alle Teilnehmerinnen erhielten keine Hormonersatztherapie.

Bereits nach vierwöchiger Applikation des Prüfpräparates wurde bei $98 \%$ der Teilnehmerinnen mittels DensiScore ${ }^{\circledR}$ eine signifikante Besserung des Hautfaltenscores am behandelten Arm ermittelt. Der Score sank hier um 24\% (Abnahme um 1,1 Punkte im Vergleich zum Ausgangsscore), während er am unbehandelten Arm um 0,4 Punkte (4\%) anstieg. Der Unterschied zwischen behandelter und unbehandelter Haut war mit einem p-Wert von $<0,001$ statistisch signifikant. Darüber hinaus ergab die klinische Beurteilung mit Hilfe einer fotografischen Skala zur Bewertung der Hauttrockenheit eine deutliche Besserung dieses Parameters im Studienverlauf. Im Vergleich zum Ausgangsbefund sank der Score bis zur vierten Behandlungswoche um 93\% ( $p<0,001) .36$ der 45 Teilnehmerinnen besaßen bei Studienende eine normale Haut mit einem Scorewert von 0. Die tägliche Anwendung der sojaextrakthaltigen Körperlotion führt somit nach Aussage der Autoren zu einer signifikanten Besserung von Dichte und Trockenheit postmenopausaler Körperhaut.

\section{Ausblick}

Die beiden vorgestellten Studien sprechen für eine positive Entwicklung in der Kosmetikindustrie, die sich für kontrollierte Studien geöffnet hat. Dieser Weg sollte konsequent weiter verfolgt werden. Neben der Wahl eines methodisch einwandfreien Studiendesigns mit multizentrischem, randomisiertem und kontrolliertem Protokoll sind außerdem instrumentalisierte Messungen unverzichtbar, um reproduzierbare Aussagen zu erhalten. Hier sind zukünftig weitere Fortschritte zu erwarten, wenn neue Techniken wie Laser-Scanning-Mikroskopie, optische Kohärenztomografie oder hoch auflösende Ultraschallgeräte zur Darstellung des Faltenreliefs zur Verfügung stehen.

\section{Literatur}

${ }^{1}$ Griffiths CE. The role of retinoids in the prevention and repair of aged and photoaged skin. Clin Exp Dermatol 2001 Oct ; 26(7): 613-618

2 Varani J, Warner RL, Gharaee-Kermani M, Phan SH, Kang S, Chung JH, Wang ZQ Datta SC, Fisher GJ, Voorhees JJ. Vitamin A antagonizes decreased cell growth and elevated collagen-degrading matrix metalloproteinases and stimulates collagen accumulation in naturally aged human skin. J Invest Dermatol 2000 Mar ; 114(3): 480-486

${ }^{3}$ Kockaert M, Neumann M. Systemic and topical drugs for aging skin. J Drugs Dermatol 2003; 2 (4): 435-441

4 Pinnell SR. Cutaneous photodamage, oxidative stress, and topical antioxidant protection. J Am Acad Dermatol 2003 Jan ; 48(1): 1 - 19

${ }^{5}$ Ruiz-Larrea MB, Mohan AR, Paganga G, Miller NJ, Bolwell GP, RiceEvans CA. Antioxidant Activity of Phytoestrogenic Isoflavones. Free Rad Res 1996; 26: $63-70$

${ }^{6}$ Spinozzi F, Pagliacci MC, Migliorati G, Moraca R, Grignani F, Riccardi C, Nicoletti I. The natural tyrosine kinase inhibitor genistein produces cell cycle arrest and apoptosis in Jurkat T-leukemia cells. Leuk Res. 1994 Jun; 18(6): 431 - 439

${ }^{7}$ Punnonen R. Effect of castration and peroral estrogen therapy on the skin. Acta Obstet Gynecol Scand Suppl 1972; 21: 3-44

8 Daten von L'Oréal Recherche

${ }^{9}$ Noch unpublizierte Daten von Vichy Cosmétique Active Deutschland

${ }^{10}$ Bayerl C, Keil D. Isoflavonoide in der Behandlung der Hautalterung postmenopausaler Frauen. Akt Dermatol 2002; 28: S14-S18

11 Bazin R, Pozzo di Borgo F, Bouloc A, Abella ML, Hirt JP. DensiScore ${ }^{\circledR}$ : a new tool for clinical evaluation of the age dependent mechanical properties of female skin. Ann Dermatol Venerol 2002; 129: 1S3711 S602

${ }^{12}$ Sparavigna A, Setaro M, Segot E, Bouloc A. Assessement of the Efficacy of a body lotion containing isoflavone for postmenopausal women. Poster. EADV, Barcelona: 2003 